\title{
ON DENSE EMBEDDINGS INTO MOORE SPACES WITH THE BAIRE PROPERTY
}

\author{
DAVID L. FEARNLEY ${ }^{\bowtie}$ and LAWRENCE FEARNLEY
}

(Received 14 June 2009)

\begin{abstract}
We demonstrate a construction that will densely embed a Moore space into a Moore space with the Baire property when this is possible. We also show how this technique generates a new 'if and only if' condition for determining when Moore spaces can be densely embedded in Moore spaces with the Baire property, and briefly discuss how this condition can can be used to generate new proofs that certain Moore spaces cannot be densely embedded in Moore spaces with the Baire property.
\end{abstract}

2000 Mathematics subject classification: primary 54E30; secondary 54B05, 54H05.

Keywords and phrases: Moore space, Baire.

\section{Introduction}

For any open covering $C$ of a space $S$, we define the star of a set $E$ in $C$, denoted $\operatorname{st}(E, C)$, to be the union of all elements of $C$ which intersect $E$. A development $G$ for a space $S$ is a sequence $G_{1}, G_{2}, G_{3}, \ldots$ of open coverings of $S$ such that for every open set $U$ and point $p \in U$ there is a positive integer $n$ such that $\operatorname{st}\left(p, G_{n}\right) \subset U$. We will also require that $G_{n+1} \subset G_{n}$ for all $n$. We say that $S$ is developable if $S$ has a development. A Moore space $S$ is a regular developable Hausdorff space.

A $\pi$-base for a space $S$ is a collection $C$ of open sets such that for every open set $U$ in $S$ there is an open set $V \in C$ such that $V \subset U$. A $\pi$-base $C$ is $\sigma$-discrete if $C$ is a countable union of discrete collections of open sets.

Reed [5] asked whether every Moore space with a $\sigma$-discrete $\pi$-base can be densely embedded in any Moore space with the Baire property. This question was answered negatively in [2].

In this paper we present some constructions that may help identify which Moore spaces can be densely embedded into a Moore space with the Baire property, and generate a more general method for constructing Moore spaces with $\sigma$-discrete $\pi$-bases that cannot be densely embedded into a Moore space with the Baire property.

From Moore's original axioms [4], a Moore space $S$ is said to satisfy axiom $\mathrm{C}$ at a point $p \in S$ with respect to a development $G=\left(G_{1}, G_{2}, G_{3}, \ldots\right)$ if for every open

(C) 2010 Australian Mathematical Publishing Association Inc. 0004-9727/2010 \$16.00 
set $U$ containing $p$ there is a positive integer $n$ such that for every $g_{n} \in G_{n}$, if $p \in g_{n}$ then $\operatorname{st}\left(g_{n}, G_{n}\right) \subset U$. Moore proved that a Moore space $S$ is metrizable if and only if there is a development for $S$ which satisfies axiom $C$ at every point of $S$.

We define a development $G$ to be a star $\pi$-base development if for every open set $U$ there is some $g_{n} \in G_{n}$ such that $\operatorname{st}\left(g_{n}, G_{n}\right) \subset U$.

Let $S$ be a Moore space with a development $G$. A sequence of development members $g_{n_{1}}, g_{n_{2}}, g_{n_{3}}, \ldots$ satisfying the conditions $g_{n_{i}} \in G_{n_{i}}, \operatorname{st}\left(g_{n_{i+1}}, G_{n_{i+1}}\right) \subset g_{n_{i}}$, $\bigcap_{i=1}^{\infty} g_{n_{i}}=\emptyset$ and $n_{i+1}>n_{i}$, for all positive integers $i$, will be called a star nested sequence for $G$. If we omit the condition that the intersection is empty we still say that the stars of the development members in the sequence are nested, but do not refer to the sequence as a 'star nested sequence'. A star nested sequence $\left(g_{n_{i}}\right)$ is defined to be equivalent to a star nested sequence $\left(g_{m_{i}}\right)$ if each member of each of these sequences contains some member of the other.

Let $M$ be the set of all equivalence classes $\left[\left(g_{n_{i}}\right)\right]$ of star nested sequences for $G$. Then we define $C(S, G)=S \cup M$. For every open set $U \subset S$ we define

$$
U^{*}=U \cup\left\{\left[\left(g_{n_{i}}\right)\right] \in M \mid g_{n_{j}} \subset U \text { for some positive integer } j\right\} .
$$

We define the topology on $C(S, G)$ to be the set of all such sets $U^{*}$. It is straightforward to verify that this is a valid topology for $C(S, G)$, and is independent of the choice of star nested sequence used in each equivalence class. It is also helpful to notice at this point that whenever $U$ and $V$ are disjoint open sets, the sets $U^{*}$ and $V^{*}$ are also disjoint. Similarly, it is helpful to see that if $U \subset V$ then $U^{*} \subset V^{*}$. Also notice that $S$ is dense in $C(S, G)$.

In this construction, whenever $G$ is a star $\pi$-base development, the space $C(S, G)$ will be shown not only to be a developable Hausdorff space with the Baire property, but also to be a space which necessarily contains a Moore space with the Baire property into which $S$ can be densely embedded, if $S$ can be densely embedded into any Moore space with the Baire property. We show that $S$ has a $\sigma$-discrete $\pi$-base if and only if $S$ has star $\pi$-base development.

\section{Embedding Moore spaces into Moore spaces with the Baire property}

THEOREM 2.1. A necessary and sufficient condition for a Moore space $S$ to have a $\sigma$-discrete $\pi$-base is that $S$ has a star $\pi$-base development.

PROOF. Necessity follows from the fact that a Moore space $S$ has a $\sigma$-discrete $\pi$-base if and only if $S$ has a development satisfying axiom $\mathrm{C}$ at a dense subset, proven by Fitzpatrick [3].

We wish to show sufficiency. Our approach will be to start with maximal disjoint collections of development members, and then within these create a $\pi$-base from a countable set of collections of development members with disjoint stars. Since a collection of development open sets whose stars are disjoint in some $G_{i}$ will necessarily be a discrete collection of open sets, this will give us the desired $\pi$-base. 
Let $S$ be a Moore space which has a star $\pi$-base development $G=\left(G_{1}, G_{2}, \ldots\right)$. Well-order $G_{i}$ for each positive integer $i$. With respect to this well ordering we define a subcollection of $G_{i}$ with induced well ordering as follows. Define $g(i, 1)$ to be the first member of $G_{i}$ and, if we have defined $g(i, \alpha)$ for $\alpha<\beta$, define $g(i, \beta)$ to be the first member of $G_{i}$ that is disjoint from each $g(i, \alpha), \alpha<\beta$, if any such member of $G_{i}$ exists. Denote by $H_{i}$ the set of all members of this generalized subsequence of $G_{i}$.

For every pair $i, j$ of positive integers we construct a set $D(i, j)$ as follows. For each $g(i, \alpha) \in H_{i}$ we choose some $g_{j} \in G_{j}$ so that $\operatorname{st}\left(g_{j}, G_{j}\right) \subset g(i, \alpha)$ if any such $g_{j}$ exists. Let $D(i, j)$ be the set of all $g_{j}$ thus chosen. Note that for every $g(i, \alpha)$ there is some $j$ such that $D(i, j)$ contains some $g_{j} \in g(i, \alpha)$.

To show that each $D(i, j)$ is discrete, let $x \in S$ and let $x \in g \in G_{j}$. If $g$ intersects any member $g_{j}$ of $D(i, j)$ then $g$ must be a subset of the star of $g_{j}$, and hence must be contained entirely in some $g(i, \alpha) \in H_{i}$. But the members of $H_{i}$ are disjoint. Thus $g$ intersects only one member of $D(i, j)$, so $D(i, j)$ is discrete.

The proof of sufficiency will be complete if we can show that

$$
D=\bigcup_{i, j \in \mathbf{N}} D(i, j)
$$

is a $\pi$-base for $S$. To do this, let $U$ be any open set of the topology of $S$. Since the development $G$ is a star $\pi$-base development, there exist an integer $k$ and a $g_{k} \in G_{k}$ such that $\operatorname{st}\left(g_{k}, G_{k}\right) \subset U$. But, from the maximal nature of the construction of $H_{k}$, we see that $g_{k}$ must intersect an element $g(k, \alpha)$ of $H_{k}$, and therefore $g(k, \alpha) \subset U$. We know there exists an integer $n$ such that for some element $g_{n} \in G_{n}, \operatorname{st}\left(g_{n}, G_{n}\right) \subset$ $g(k, \alpha)$, so then there exists some element of $D(k, n)$ which is a subset of $U$.

Reed [5] proved that every Moore space can be densely embedded in a developable Hausdorff space. We do not assert that the following construction has an advantage over Reed's original construction for purposes of proving that result. However, we wish to show specifically that $C(S, G)$ is a developable quasi-regular Hausdorff space with the Baire property since we will use this fact later. A space $S$ is said to be quasiregular if for every open set $U \subset S$ there is an open set $V$ such that $\bar{V} \subset U$.

THEOREM 2.2. If $S$ is a Moore space having a star $\pi$-base development $G$ then $C(S, G)$ is a quasi-regular Hausdorff developable space having the Baire property.

ProOf. Let $G=\left(G_{1}, G_{2}, G_{3}, \ldots\right)$ be a star $\pi$-base development for $S$. For each positive integer $i$, we define $H_{i}=\left\{g_{i}^{*} \mid g_{i} \in G_{i}\right\}$. We claim that $H=$ $\left(H_{1}, H_{2}, H_{3}, \ldots\right)$ is a development for $C(S, G)$. To see this, let $p \in U^{*}$, an open set in $C(S, G)$.

If $p \in S$ then choose $n$ so that $\operatorname{st}\left(p, G_{n}\right) \subset U$. Then $\operatorname{st}\left(p, H_{n}\right) \subset U^{*}$, since if $p \in g_{n}^{*}$ then $p \in g_{n}$ and therefore $g_{n} \subset U$, so $g_{n}^{*} \subset U^{*}$. If $p=\left[\left(g_{m_{i}}\right)\right]$ then for any $i>n$ it must follow that $\operatorname{st}\left(g_{m_{i}}, G_{m_{i}}\right) \subset U$ and therefore $\operatorname{st}\left(p, H_{n}\right) \subset U$.

We wish to show that $C(S, G)$ is quasi-regular. Let $U^{*}$ be an open set in $C(S, G)$. Since $G$ is a star $\pi$-base development there is some $g_{n} \in G_{n}$ such that $\operatorname{st}\left(g_{n}, G_{n}\right) \subset U$. 
Then $g_{n}^{*}$ is open and $\overline{g_{n}^{*}} \subset U^{*}$. To show that $C(S, G)$ is Hausdorff we consider three cases.

First, suppose that $x, y \in S$. Then for disjoint open sets $U$ and $V$ containing $x$ and $y$ respectively, $U^{*}$ and $V^{*}$ are also disjoint.

Next, suppose that $x, y \in M$, where $x=\left[\left(g_{m_{i}}\right)\right]$ and $y=\left[\left(g_{n_{i}}\right)\right]$. Since $x \neq y$ we can choose $j$ so that $g_{m_{j}}$ contains no $g_{n_{i}}$ and $k$ so that $g_{n_{k}}$ contains no $g_{m_{i}}$. But then $g_{m_{j+1}}$ and $g_{n_{k+1}}$ are disjoint by the star nested property (for instance, if $m_{j+1}>n_{k+1}$ then $g_{m_{j+1}} \cap g_{n_{k+1}} \neq \emptyset$ would imply $g_{m_{j+1}} \subset g_{n_{k}}$ ). Thus, $g_{m_{j+1}}^{*}$ and $g_{n_{k+1}}^{*}$ are disjoint open sets containing $x$ and $y$.

Finally, suppose that $x \in S$ and $y \in M$. Then let $y=\left[\left(g_{n_{i}}\right)\right]$. Choose $k$ so that $x \notin g_{n_{k}}$. By star nesting, if we let $x \in g \in G_{n_{k+1}}$, it follows that $g \cap g_{n_{k+1}}=\emptyset$ and therefore $g^{*} \cap g_{n_{k+1}}^{*}=\emptyset$.

Finally, we show that $C(S, G)$ has the Baire property. Let $U^{*}$ be an arbitrary open set in $C(S, G)$ and let $D_{1}^{*}, D_{2}^{*}, D_{3}^{*}, \ldots$ be a sequence of dense open sets in $C(S, G)$. Since $D_{1}^{*}$ is a dense open set and $U^{*}$ is a nonempty open set, $U^{*} \cap D_{1}^{*}$ is a nonempty open set in $C(S, G)$. Hence, there exist an integer $n_{1}$ and a member $g_{n_{1}}$ of $G_{n_{1}}$ such that $\operatorname{st}\left(g_{n_{1}}, G_{n_{1}}\right) \subset\left(U \cap D_{1}\right)$. Next, $g_{n(1)}^{*}$ intersects $D_{2}^{*}$ so there is an integer $n_{2}$, with $n_{2}>n_{1}$, such that $\operatorname{st}\left(g_{n_{2}}, G_{n_{2}}\right) \subset\left(g_{n_{1}} \cap D_{2}\right)$. Proceeding in this manner, we note that if there is no element of $S$ in the intersection of the $g_{n_{i}}$ then $\left[\left(g_{n_{i}}\right)\right] \in M$, and so either $U^{*} \cap D_{1}^{*} \cap D_{2}^{*} \cap D_{3}^{*} \cap \cdots$ contains a point of $S$ or it contains the point $\left[\left(g_{n_{i}}\right)\right]$. Thus $C(S, G)$ has the Baire property.

We note at this point that just because a Moore space has a star $\pi$-base development, that does not imply that this development satisfies axiom $\mathrm{C}$. The following example is a subspace of an example due to Bing (it is part of Bing's A), which was used for a different purpose in his paper. Bing presented his example A as an example of a Moore space which has no open metrizable subsets [1].

EXAMPLE 2.3. A Moore space $S$ with a star $\pi$-base development $G$ such that $G$ does not satisfy axiom $C$ at any point of $S$.

Proof. Bing's A, which we denote by $A$, can be constructed by letting the points of $A$ be points of the plane, and defining the topology by a development $W=$ $\left(W_{1}, W_{2}, W_{3}, \ldots\right)$ as follows. Let $H_{1}, H_{2}, \ldots$ be the horizontal lines in the plane with rational height. We let $W_{n}$ consist of all open balls in the plane of diameter less than $1 / n$ which do not intersect $\bigcup_{i=1}^{n} H_{i}$, plus all of the unions $D_{U} \cup D_{B} \cup\{p\}$, where $p$ is a point of some horizontal line $H_{m}$ so that $m \leq n$ and $D_{U}, D_{B}$ are open disks in the plane of diameter less than $1 / n$, each tangent to $H_{m}$ at the point $p$, with $D_{U}$ above $p$ and $D_{B}$ below $p$.

The space $S$ is a subspace of $A$ consisting of only the horizontal lines $H_{i}$. We let $G$ be the development for $S$ induced by $W$. That is, for each $w_{n} \in W_{n}$ we let $g_{n}=w_{n} \cap S$.

This example is a Moore space with a $\sigma$-discrete $\pi$-base for which the given development $G$ fails to satisfy axiom $\mathrm{C}$ at any point with respect to the given 
development, even though $G$ is a star $\pi$-base development for $S$. The fact that the space $S$ is Moore follows from the fact that it is a subspace of a Moore space. We must check that $G$ is a development satisfying axiom $C$ at no point and is a star $\pi$-base development. It is straightforward to see that $G$ is a development.

To show that $G$ does not satisfy axiom $\mathrm{C}$ at any point, let $p \in H_{m}$ and choose $V \in$ $G_{m+1}$ so that $p \in V$. Let $p \in g_{n} \subset V$ for some $g_{n} \in G_{n}$, where $n>m$. Let $q \in H_{m}$ which has distance from $p$ less than the minimum of $\{1 / n\} \cup\left\{d\left(H_{i}, H_{j}\right) \mid i, j \leq n\right\}$. Then by construction of $G_{n}$ we can see that $q$ is an element of $\operatorname{st}\left(g_{n}, G_{n}\right)$. But $q \notin V$ so $G$ does not satisfy axiom $\mathrm{C}$ at any point.

Finally, to show that $G$ is a star $\pi$-base development, let $U$ be an open set so that the planar open disk $B_{1 / m}(x) \cap S \subset U$ and choose a point $p \in B_{1 / n}(x) \cap S$ for some $n>4 m$. Let $p \in g_{n} \in G_{n}$. Then $\operatorname{st}\left(g_{n}, G_{n}\right) \subset U$.

THEOREM 2.4. If a Moore space $S$ with a $\sigma$-discrete $\pi$-base can be densely embedded in a Moore space with the Baire property, then for some star $\pi$-base development $G$, there is a Moore subspace $X$ of $C(S, G)$ which contains $S$ and has the Baire property.

PROOF. Assume that $S$ can be densely embedded in a Moore space $T$ with the Baire property. Then $T$ has a development $W$ satisfying axiom $\mathrm{C}$ at all points of a dense subset (Reed [5]). Then intersecting the elements of the members of $W$ with $S$ gives a star $\pi$-base development $G$ of $S$, where $G_{n}=\left\{w \cap S \mid w \in W_{n}\right\}$.

By way of description, we wish to choose maximal collections $H_{n}$ of development members with stars nested in previous stages. More specifically, we are picking a subset of $G_{1}$ with enough elements so that the union will be dense. Then we keep picking, in every open set we chose in the previous stage, higher indexed development members whose stars are contained in that open set, until we have filled up the union of the previous stage enough to attain another dense subset of $S$. Everything in the intersection of the unions of the stages is then the intersection of a sequence of development members whose stars are nested.

First, well-order the elements of $G_{1}$ as sets $g(1, \alpha)$. Then let $g(1,1)=h(1,1) \in$ $H_{1}$. If we have chosen $h(1, \alpha)$ for all $\alpha<\beta$ then we let $h(1, \beta)$ be the first element of $G_{1}$ that does not intersect $\bigcup_{\alpha<\beta} h(1, \alpha)$ if any such set exists. Then $H_{1}$ is the set of all such $h(1, \alpha)$. Then, if we have chosen $H_{n}$, we well-order all of the elements of $\bigcup G$. We let $g(n, \alpha) \in G_{n}$ denote the $\alpha$ th indexed member of the ordering. Let $h(n+1,1)=g\left(m, \alpha_{1}\right)$ where $\alpha_{1}$ is the first index so that for some $h(n, \gamma) \in$ $H_{n} \cap G_{k}, m>k$ and $\operatorname{st}\left(g\left(m, \alpha_{1}\right), G_{m}\right) \subset h(n, \gamma)$. If we have chosen $h(n+1, \alpha)$ for all $\alpha<\beta$ then we let $h(n+1, \beta)=g\left(m_{\beta}, \alpha_{\beta}\right)$, where $g\left(m_{\beta}, \alpha_{\beta}\right)$ is the first element of $\bigcup G$ that does not intersect any $h(n+1, \alpha)$ for any $\alpha<\beta$, having the property that for some $h(n, \gamma) \in H_{n} \cap G_{k_{\beta}}, m_{\beta}>k_{\beta}$ and $\operatorname{st}\left(g\left(m_{\beta}, \alpha_{\beta}\right), G_{m_{\beta}}\right) \subset h(n, \gamma)$. The set of all $h(n+1, \alpha)$ thus chosen is $H_{n+1}$.

Note that each $\bigcup H_{n}$ is open and dense in $S$. For each open set $U$ in $S$, we define $U^{T}$ to be the open set in $T$ consisting of the union of all open subsets of $T$ whose intersection with $S$ is contained in $U$. Since $T$ has the Baire property and $D_{n}=\bigcup H_{n}^{T}$ 
is open and dense in $T$ for all $n$, it follows that $D=\bigcap_{n=1}^{\infty} D_{n}$ is a dense $G_{\delta}$ subset of $T$.

For every point $x \in D$ there is a sequence of development members $\left(g\left(n_{k}, \alpha_{k}\right)_{x}\right)$ so that the $\left(n_{k}\right)$ are increasing, $x \in g\left(n_{k}, \alpha_{k}\right)_{x}^{T}$, and

$$
\operatorname{st}\left(g\left(n_{k+1}, \alpha_{k+1}\right)_{x}, G_{n_{k+1}}\right) \subset g\left(n_{k}, \alpha_{k}\right)_{x}
$$

for all positive integers $k$. Notice also that $x=\bigcap_{n=1}^{\infty} g\left(n_{k}, \alpha_{k}\right)_{x}^{T}$. This is because for any $y \neq x$ we can find $t$ so that if $U, V \in W_{t}$ and $x \in U$ and $y \in V$ then $U \cap V=\emptyset$. Therefore, no member of $W_{t}$ containing $y$ can intersect $g\left(n_{t}, \alpha_{t}\right)$.

We will let $X$ be the subset of $C(S, G)$ consisting of $S$ plus all star nested sequences $\left[\left(g\left(n_{k}, \alpha_{k}\right)\right)\right]$ such that $g\left(n_{k}, \alpha_{k}\right) \in H_{k}$ for all positive integers $k$, and there is some point $x \in T$ such that $x=\bigcap_{n=1}^{\infty} g\left(n_{k}, \alpha_{k}\right)^{T}$. Since $x$ is uniquely associated with the equivalence class $\left[\left(g\left(n_{k}, \alpha_{k}\right)\right)\right]$, it is convenient to write this class as $\left[\left(g\left(n_{k}, \alpha_{k}\right)_{x}\right)\right]$. We define a function $f: X \rightarrow T$ by letting $f(s)=s$ if $s \in S$ and $f\left(\left[\left(g\left(n_{k}, \alpha_{k}\right)_{x}\right)\right]\right)=x$ otherwise.

We know that any subset of a Baire space containing a dense $G_{\delta}$ subset of that space is itself Baire. Thus, we need only show that $f$ is a homeomorphism and $D \subset f(X)$. By construction, if $x \in D$ then there is a sequence $\left(g\left(n_{k}, \alpha_{k}\right)_{x}\right)$ such that $\bigcap_{k=1}^{\infty} g\left(n_{k}, \alpha_{k}\right)_{x}^{T}=x$. Hence, either $x \in S$ or $\left[\left(g\left(n_{k}, \alpha_{k}\right)_{x}\right)\right] \in(M \cap X)$. Thus, $D \subset f(X)$.

It is clear that $f$ is bijective on $S$, and we have also established that the association between star nested sequences of $X$ and points of $T$ is unique. Thus, $f$ is bijective.

Let $U$ be open in $f(X)$. Then $U=V \cap f(X)$ for some open $V$ in $T$. Hence, $f^{-1}(U) \cap S=V \cap S$. Also $x \in U \backslash S$ if and only if, for some positive integer $i$, it is the case that $\operatorname{st}\left(g\left(n_{i}, \alpha_{i}\right)_{x}, G_{i}\right) \subset U \cap S$, which is true if and only if $\left[\left(g\left(n_{k}, \alpha_{k}\right)_{x}\right)\right] \in$ $f^{-1}(U)$. Thus, $f^{-1}(U)=U^{*} \cap X$, and hence $f$ is a homeomorphism.

We can extend this somewhat to show that the particular choice of star $\pi$-base development is unimportant. This extension is quite valuable, because it means that we can take any Moore space $S$, pick any convenient star $\pi$-base development whose star nested sequences will generate a space we find easy to work with, and just determine whether adding enough points to that particular space can make a superspace Baire without violating regularity.

THEOREM 2.5. If a Moore space $S$ can be densely embedded in a Moore space with the Baire property, and $G$ is any star $\pi$-base development for $S$ then there is a subspace of $C(S, G)$ which is a Moore space with the Baire property and contains $S$.

Proof. By the previous theorem, we may choose a star $\pi$-base development $G^{\prime}$ for $S$ and a subspace $X^{\prime} \subset C\left(S, G^{\prime}\right)=S \cup M^{\prime}$ so that $X^{\prime}$ is a Moore space with the Baire property containing $S$. Let $C(S, G)=S \cup M$ as usual, and let

$$
\begin{gathered}
X=S \cup\left\{\left[\left(g_{n_{i}}\right)\right] \in M \mid \exists\left[\left(g_{n_{i}}^{\prime}\right)\right] \in M^{\prime} \text { such that } \forall m \exists k>m\right. \\
\text { so that } \left.g_{n_{k}} \subset g_{n_{m}}^{\prime} \text { and } g_{n_{k}}^{\prime} \subset g_{n_{m}}\right\} .
\end{gathered}
$$


Essentially, $X$ is constructed by taking all the star nested sequences in $C(S, G)$ that are equivalent to star nested sequences in $X^{\prime}$.

We note that there is no difference between the topology on $X$ induced as a subspace of $C(S, G)$ and the topology on $X$ as a subspace of $X^{\prime}$ (where equivalent sequences in the two developments are considered to be the same point of both spaces) because open sets in $S$ containing a member of a star nested sequence in the $G$ development will always contain a member of an equivalent star nested sequence in the $G^{\prime}$ development and vice versa. Then we claim that $X$ is a Moore space with the Baire property which contains $S$. That $X$ is a Moore space follows from the fact that $X \subset X^{\prime}$.

It remains to be shown that $X$ has the Baire property. Our strategy is to proceed in a manner similar to the construction of the $H_{i}$ in the preceding theorem, except that we will alternate between the developments $G$ and $G^{\prime}$. This will create a dense $G_{\delta}$ subset of $X^{\prime}$ which is also a subset of $X$, and that will complete the proof. Since there are two developments being used to generate $C(S, G)$ spaces in this theorem, to avoid ambiguity, for an open set $U$ in $S$ we will let $U^{*}$ be the usual definition of $U^{*}$ with reference to the development $G$, and we will let $U^{\prime *}$ be the usual open set of $X^{\prime}$ corresponding to $U$ with reference to the development $G^{\prime}$.

First, well-order the elements of $G_{1}$ as sets $g(1, \alpha)$. Then let $g(1,1)=h(1,1) \in$ $H_{1}$. If we have chosen $h(1, \alpha)$ for all $\alpha<\beta$ then we let $h(1, \beta)$ be the first element of $G_{1}$ that does not intersect $\bigcup_{\alpha<\beta} h(1, \alpha)$ if any such set exists. Then $H_{1}$ is the set of all such $h(1, \alpha)$. Well-order all elements of $\bigcup G$ and $\bigcup G^{\prime}$. We let $g(n, \alpha) \in G_{n}$ denote the $\alpha$ th indexed member of the ordering on $\bigcup G$, and similarly we let $g(n, \alpha)^{\prime} \in G_{n}^{\prime}$ denote the $\alpha$ th indexed member of the ordering on $\bigcup G^{\prime}$. Then, if we have chosen $H_{n}$ and $n$ is odd, we let $h(n+1,1)=g\left(m, \alpha_{1}\right)^{\prime}$ where $\alpha_{1}$ is the first index so that for some $h(n, \gamma) \in H_{n} \cap G_{k}^{\prime}, m>k$ and $\operatorname{st}\left(g\left(m, \alpha_{1}\right)^{\prime}, G_{m}^{\prime}\right) \subset h(n, \gamma)$. If we have chosen $h(n+1, \alpha)$ for all $\alpha<\beta$ then we let $h(n+1, \beta)=g\left(m_{\beta}, \alpha_{\beta}\right)^{\prime}$, where $g\left(m_{\beta}, \alpha_{\beta}\right)^{\prime}$ is the first element of $\bigcup G^{\prime}$ that does not intersect any $h(n+1, \alpha)$ for any $\alpha<\beta$, having the property that for some $h(n, \gamma) \in H_{n} \cap G_{k_{\beta}}^{\prime}, m_{\beta}>k_{\beta}$ and $\operatorname{st}\left(g\left(m_{\beta}, \alpha_{\beta}\right)^{\prime}, G_{m_{\beta}}^{\prime}\right) \subset h(n, \gamma)$. The set of all $h(n+1, \alpha)$ thus chosen is $H_{n+1}$.

Similarly, if $n$ is even then we let $h(n+1,1)=g\left(m, \alpha_{1}\right)$ where $\alpha_{1}$ is the first index so that for some $h(n, \gamma) \in H_{n} \cap G_{k}, m>k$ and $\operatorname{st}\left(g\left(m, \alpha_{1}\right), G_{m}\right) \subset h(n, \gamma)$. If we have chosen $h(n+1, \alpha)$ for all $\alpha<\beta$ then we let $h(n+1, \beta)=g\left(m_{\beta}, \alpha_{\beta}\right)$, where $g\left(m_{\beta}, \alpha_{\beta}\right)$ is the first element of $\bigcup G$ that does not intersect any $h(n+1, \alpha)$ for any $\alpha<\beta$, having the property that for some $h(n, \gamma) \in H_{n} \cap G_{k_{\beta}}, m_{\beta}>k_{\beta}$ and $\operatorname{st}\left(g\left(m_{\beta}, \alpha_{\beta}\right), G_{m_{\beta}}\right) \subset h(n, \gamma)$. The set of all $h(n+1, \alpha)$ thus chosen is $H_{n+1}$.

We let $D_{n}=\bigcup_{n=1}^{\infty} H_{n}^{\prime *}$, and let $D=\bigcap_{n=1}^{\infty} D_{n}$. Then $D$ is a dense $G_{\delta}$ subset of $X^{\prime}$. Let $x \in D$. Then by construction of $D$ there is a sequence of development members $\left(w\left(n_{k}, \alpha_{k}\right)_{x}\right)$ so that $w\left(n_{k}, \alpha_{k}\right)_{x}=g\left(n_{k}, \alpha_{k}\right)_{x} \in G_{n_{k}}$ if $k$ is odd and $w\left(n_{k}, \alpha_{k}\right)_{x}=$ $g\left(n_{k}, \alpha_{k}\right)_{x}^{\prime} \in G_{n_{k}}^{\prime}$ if $k$ is even, so that the $\left(n_{k}\right)$ are increasing, $x \in w\left(n_{k}, \alpha_{k}\right)_{x}^{\prime *}$ and $\operatorname{st}\left(g\left(n_{k+1}, \alpha_{k+1}\right)_{x}^{\prime}, G_{n_{k+1}}^{\prime}\right) \subset g\left(n_{k}, \alpha_{k}\right)_{x}$ for all positive odd integers $k$, and $\operatorname{st}\left(g\left(n_{k+1}, \alpha_{k+1}\right)_{x}, G_{n_{k+1}}\right) \subset g\left(n_{k}, \alpha_{k}\right)_{x}^{\prime}$ for all positive even integers $k$. Hence, since the sequences $\left(g\left(n_{2 k+1}, \alpha_{2 k+1}\right)_{x}\right)$ have nested stars, either $x \in S$ or $x \in M$. 
Thus, $D \subset C(S, G)$. Hence, it follows that $X$ contains a dense $G_{\delta}$ Baire subset, and therefore $X$ has the Baire property.

We have established a new 'if and only if' condition for such embeddings, listed here as a corollary.

Corollary 2.6. Let $X$ be a Moore space. Then $X$ can be densely embedded in a Moore space with the Baire property if and only if $X$ has a star $\pi$-base development $G$, and it is also true that for any such star $\pi$-base development $G$, some subset of $C(S, G)$ is regular and Baire.

Thus, one way of showing that a Moore space cannot be densely embedded in a Moore space with the Baire property is to find a star $\pi$-base development for $S$ so that if enough star nested sequences are made points to make $S$ Baire then the space can no longer be regular. It would seem inappropriate to reproduce the proof of the example in [2] here, but it is evident that if we just use the fact that a Baire space must be obtainable by merely turning certain star nested sequences into points then you are able to skip a large section of the proof dealing with where points would be forced to be situated in the new superspace. The example in that paper contains copies of the rational numbers. By choosing the right development we can see that adding star nested sequences as points will simply generate a subspace homeomorphic to a subset of the real numbers.

Once that has been established we would know that if the space could be embedded in a Moore space with the Baire property then there would have to be a way to add enough irrationals to each of the copies of the rational numbers to make each copy have the Baire property. One merely shows that to be impossible without violating regularity on the space and the shorter proof is finished. It is possible to use this method to find other Moore spaces that cannot be densely embedded in a Moore space with the Baire property as well.

Among other things, the following useful fact is true of Moore spaces that can be densely embedded into a Moore space with the Baire property.

THEOREM 2.7. Let $S$ be a Moore space that can be densely embedded into a Moore space with the Baire property and contains an open metrizable subset $O$. Let $W$ be any complete metric space into which $O$ can be densely embedded. Then $S$ can be densely embedded into a Moore space $X$ which has the Baire property so that the image of $O$ is contained in a subset of $X$ which is homeomorphic to a Baire subset of $W$.

Proof. Let $G$ be a star $\pi$-base development for $S$. Considering $O$ as a subset of $W$, using a metric with respect to which $W$ is complete, we let $H_{i}$ be the set of all open balls $\left\{B_{1 / i}(x) \cap O \mid x \in W\right\}$. Let $G^{\prime}=\left(G_{i}^{\prime}\right)$ be a development for $S$ obtained by letting

$$
G_{i}^{\prime}=\left\{g_{i} \in G_{i} \mid g_{i} \cap\left(\bigcup H_{i}\right)=\emptyset\right\} \cup\left\{g_{i} \cap h_{i} \mid g_{i} \in G_{i} \text { and } h_{i} \in H_{i}\right\}
$$

for every positive integer $i$. 
All elements of $G_{i}^{\prime}$ are subsets of elements of $G_{i}$, so $G_{i}^{\prime}$ is a star $\pi$-base development.

Hence, by Theorem 2.5 there is some subset $X$ of $C\left(S, G^{\prime}\right)$ that is a Moore space and has the Baire property and contains $S$. For any open $U$ in $S$, let $U_{X}^{*}$ denote the portion of $U^{*}$ that is contained in $X$. We claim that $O_{X}^{*}$ is metrizable, homeomorphic to a dense subset of $W$, and has the Baire property. It is immediate that $O_{X}^{*}$ has the Baire property since it is an open subset of a Baire space, and likewise density is clear. What remains to be shown is that $O_{X}^{*}$ is actually homeomorphic to a subset of $W$.

For every $p \in W$ and positive integer $n$ we can find an open set $g_{2^{n}}(p)^{\prime}=$ $B_{1 / 2^{n}}(p) \cap g_{2^{n}}$ containing $p$. Note that because $B_{1 / 2^{n}}(p)$ is an open subset of $W$, it follows that $g_{2^{n}}(p)^{\prime} \subset O$. Also, since the radii of the metric balls are halved with each successive sequence member, it follows that if $p \in W \backslash O$ then each $\left(g_{2^{n}}(p)^{\prime}\right)$ is a star nested sequence.

Let $\left[\left(g_{n_{k}}^{\prime}\right)\right]$ be any star nested sequence point of $O^{*}$. Then for some $m$, if $k>m$, then $g_{n_{k}}{ }_{k} \subset O$. Likewise, by the definition of $G^{\prime}$ it follows that the diameters of the sets $g_{n_{k}}{ }_{k}$, where $k>m$, are converging to zero. Hence, by completeness of $W$, if we choose a point $p_{k} \in g_{n_{k}}^{\prime}$ for all $k>m$ then we obtain a Cauchy sequence $\left(p_{k}\right)$ converging to some point $p \in W$. But then for every $k$ we can find an integer $j$ such that $\left(B_{1 / 2^{j}}(p) \cap O\right) \subset g_{n_{k}}{ }^{\prime}$ and $g_{n_{j}}{ }_{j} \subset\left(B_{1 / 2^{k}}(p) \cap O\right)$. Hence, the star nested sequences $\left(g_{n_{k}}^{\prime}\right)$ and $\left(g_{2^{n}}(p)^{\prime}\right)$ are equivalent.

We conclude that $O^{*}$ consists only of elements of $O$ and the equivalence classes $\left[\left(g_{2^{n}}(p)^{\prime}\right)\right]$, where $p \in W \backslash O$. We define $f: O^{*} \rightarrow W$ by setting $f(x)=x$ if $x \in O$ and $f(p)=\left[\left(g_{2^{n}}(p)^{\prime}\right)\right]$ otherwise. This mapping is easily seen to be bijective. The fact that $f$ is a continuous open mapping is also almost immediate. For any open set $U^{*}$ in $O^{*}$, by definition $U^{*}$ consists exactly of $U$ plus those star nested sequences whose intersection with $O$ is eventually contained in $U$. Since $O$ is dense in $W$, a sequence of open balls about a point $p \in W$ whose radii converge to zero in $O$ will eventually be contained in $U$ if and only if $p \in U$. Hence, $f$ is a homeomorphism, so $f$ restricted to any subset of $O^{*}$ is also a homeomorphism onto its image. Therefore, $O_{X}^{*}$ is homeomorphic to a subset of $W$.

We conclude with some questions.

QUESTION 2.8. Is there a separable Moore space which cannot be densely embedded in a Moore space with the Baire property?

QUESTION 2.9. Is there a Moore space $S$ that has a $\sigma$-discrete $\pi$-base such that no open subset of $S$ can be densely embedded in any Moore space with the Baire property?

\section{References}

[1] R. H. Bing, 'Metrization of topological spaces', Canad. J. Math. 3 (1951), 175-186.

[2] D. L. Fearnley, 'A Moore space with a $\sigma$-discrete $\pi$-base which cannot be densely embedded in any Moore space with the Baire property', Proc. Amer. Math. Soc. 127(10) (1999), 3095-3100. 
[3] B. Fitzpatrick, 'On dense subspaces of Moore spaces, II', Fund. Math. 61 (1967), 91-92.

[4] R. L. Moore, Foundations of Point Set Theory, American Mathematical Society Colloquium Publications, 13 (American Mathematical Society, Providence, RI, 1962), revised edition.

[5] G. M. Reed, On Completeness Conditions in Moore Spaces, Lecture Notes in Mathematics, 378 (Springer, New York, 1974), pp. 368-384.

DAVID L. FEARNLEY, Department of Mathematics, Utah Valley University, Orem, UT, USA

e-mail: axiom10@gmail.com

LAWRENCE FEARNLEY, Department of Mathematics, Brigham Young University, Provo, UT, USA

e-mail: fearnley@byu.edu 